\title{
Phase behaviour of a simple model of globular proteins
}

\author{
Richard P. Sear \\ Department of Physics, University of Surrey \\ Guildford, Surrey GU2 5XH, United Kingdom \\ email: r.sear@surrey.ac.uk
}

\begin{abstract}
A simple model of globular proteins which incorporates anisotropic attractions is proposed. It is closely related to models used to model simple hydrogen-bonding molecules such as water. Theories for both the fluid and solid phases are presented, and phase diagrams calculated. The model protein exhibits a fluidfluid transition which is metastable with respect to the fluid-solid transition for most values of the model parameters. This is behaviour often observed for globular proteins. The model offers an explanation of the difficulty observed in crystallising some globular proteins and suggests that some proteins may not have a solid phase at all under all but extreme conditions.
\end{abstract}

\section{Introduction}

The interactions between globular proteins are rather poorly understood but it seems clear that many of the attractive interactions are highly directional, -3 two protein molecules must not only be close to each other to attract each other but they must also be correctly oriented. An example is the attraction between hydrophobic patches on the surfaces of globular proteins; only if the proteins are oriented so that these parts of their surfaces face each other is there an attraction. This attraction is both highly directional and short ranged. bonds, which are highly directional, are known to have a dramatic effect on the behaviour of simple molecules such as water. For example, ice's diamond-like lattice and very low density are imposed by the tetrahedral arrangement of the hydrogen bonds formed by water molecules. It is striking that much of the language used to describe the interactions between globular proteins 13 is the same as that used in describing hydrogenbonding fluids. 6 Therefore, we believe it is very worthwhile to explore the phase behaviour of a simple model of globular proteins which includes directional attractions. We find phase diagrams of qualitatively the same form as observed in experiment. In particular, we find metastable transitions between dilute and dense fluid phases.

Solutions of globular proteins, like simple molecules, exhibit two types of phase transition: fluid-to-fluid, analogous to a vapour-liquid transition, and fluid-to-solid. The behaviour of globular proteins differs from that of simple molecules in one important respect: the transition between a dilute and a dense fluid phase is metastable with respect to solidification 7 whereas, simple molecules such as argon and water have equilibrium liquid phases. The proteins crystallise in a variety of different lattice types. This is suggestive of directional attractions as at low temperature these impose the lattice. For example, a water molecule bonds to four molecules arranged tetrahedrally around it. In a diamond lattice but not a face-centred-cubic lattice each water molecule is surrounded by four others arranged tetrahedrally, and so water freezes into a solid with a diamond not a face-centred-cubic lattice. Isotropic attractions of any range have a relatively weak effect on the solid phase; the solid is face-centred cubic as it is for hard spheres. Globular proteins are not perfectly spherical, of course and this may also have an effect on the lattice type.

Previous work 12 has ignored any directionality in the attraction and modeled the protein molecules with a potential consisting of a hard core and a short-range isotropic attraction. The phase behaviour of such a 
potential is qualitatively similar to experiment and to the behaviour we find for our model, with the exception of the solid phase. We will return to this point when we discuss our results in section 5 but the interaction between proteins almost certainly includes both isotropic and directional attractions.

The work is presented as follows. In the next section we specify the simplest model of a protein we can devise and which includes directional attractions. It does not include isotropic attractions and all the directional attractions are of the same strength. This model is very similar but not identical to very simple models of water. 1315 Given its simple nature and our lack of knowledge of the interaction between globular proteins our analysis will be purely qualitative. Nevertheless, we can describe the experimental phase behaviour and the phase diagrams we calculate have intrinsic interest as some of the first calculations which include the fluid and solid phases when the attractions are highly directional. For related work see Ref. 15. The theories for the fluid and solid phases are derived in sections 3 and 4, respectively. We restrict ourselves to a brief summary of the theory for the fluid phase as it is well established, it is based on the formalism derived by Wertheim. 1621 The theory for the solid phase is new, it is a cell theory, like that of Vega and Monson.15 Example phase diagrams are shown and discussed in section 5. We finish with a discussion of the connection between the interactions and both the fluid-fluid transition and the stability or otherwise of the solid phase.

\section{Model}

Our model is not new, it is of a type which has been widely employed to model hydrogen-bonding molecules such as water and alcohols. Indeed, the idea of a potential consisting of a repulsion plus a sticky patch goes back to Boltzmann.22 Specifically, we will consider the conical-site model of Chapman, Jackson and Gubbins.20121 A schematic of the model is shown in Fig. 1. The potential is a pair potential $\phi$ which is a sum of two parts: a hard-sphere repulsion, $\phi_{h s}$, and a set of sites which mediate short-range, directional attractions. The sites come in pairs: a site on one particle binds only to its partner on another particle. So, the number of sites, $n_{s}$, is constrained to be an even number. The two sites of a pair are numbered consecutively so that an odd-numbered site, $i$, binds only to the even-numbered site, $i+1$. This is the only interaction between the sites, an odd-numbered site, $i$, does not interact at all with sites other than the $(i+1)$ th site. Each site has a specific orientation, see Fig. 1. The orientation of site number $i$ is specified by means of a unit vector $\mathbf{u}_{i}$.

We can write the interaction potential between a pair of particles as

$$
\phi\left(r_{12}, \Omega_{1}, \Omega_{2}\right)=\phi_{h s}\left(r_{12}\right)+\sum_{i}^{\prime}\left[\phi_{i i+1}\left(r_{12}, \Omega_{1}, \Omega_{2}\right),+\phi_{i i+1}\left(r_{12}, \Omega_{2}, \Omega_{1}\right)\right],
$$

where the dash on the first sum denotes that it is restricted to odd values of $i$. The interactions between the sites on the two particles are $\phi_{i i+1}\left(r_{12}, \Omega_{1}, \Omega_{2}\right)$, which is the interaction between site $i$ on particle 1 and site $i+1$ on particle 2 , and $\phi_{i i+1}\left(r_{12}, \Omega_{2}, \Omega_{1}\right)$, which is the interaction between site $i$ on particle 2 and site $i+1$ on particle 1 . These are functions of $r_{12}, \Omega_{1}$ and $\Omega_{2}$, which are the scalar distance between the centres of particles 1 and 2, the orientation of particle 1 and the orientation of particle 2, respectively. The particle is rigid, but not axially symmetric, so its position is completely specified by the position of its centre and its orientation $\Omega$, which may be expressed in terms of the three Euler angles.23

The hard-sphere potential, $\phi_{h s}$, is given by

$$
\phi_{h s}(r)= \begin{cases}\infty & r \leq \sigma \\ 0 & r>\sigma\end{cases}
$$

where $\sigma$ is the hard-sphere diameter. The conical-site interaction potential $\phi_{i i+1}$ is given by 20

$$
\phi_{i i+1}\left(r_{12}, \Omega_{1}, \Omega_{2}\right)=\left\{\begin{array}{ll}
-\epsilon & r_{12} \leq r_{c} \\
0 & \text { otherwise }
\end{array} \text { and } \quad \theta_{1 i} \leq \theta_{c} \quad \text { and } \quad \theta_{2 i+1} \leq \theta_{c}\right.
$$

where $\theta_{1 i}$ is the angle between a line joining the centres of the two particles and the unit vector $\mathbf{u}_{i}$ of particle 1 , and $\theta_{2 i+1}$ is the angle between a line joining the centres of the two particles and the unit vector $\mathbf{u}_{i+1}$ of 
particle 2. The conical-site potential depends on two parameters: the range, $r_{c}$, and the maximum angle at which a bond is formed, $\theta_{c}$. Of course, as the attractions are directional, $\theta_{c}$ will be small, no more than about $30^{\circ}$. The attractions are also short ranged, $r_{c}$ no more than $10 \%$ larger than $\sigma$.

The angles between the site orientations, the vectors $\mathbf{u}_{i}$ will determine which solid lattice is formed. This is like water, where the diamond-like lattice is enforced by the tetrahedral arrangements of the hydrogen bonds. For simplicity, we will take the sites to be arranged such that they are compatible with a simple cubic lattice. Then if we express the unit vectors $\mathbf{u}_{i}$ in Cartesian coordinates, $(x, y, z)$, then when we have four sites, $n_{s}=4$, the set of vectors $\mathbf{u}_{1}=(1,0,0), \mathbf{u}_{2}=(-1,0,0), \mathbf{u}_{3}=(0,1,0)$ and $\mathbf{u}_{4}=(0,-1,0)$ would describe our model. For six sites then we add two additional sites at orientations $\mathbf{u}_{5}=(0,0,1)$ and $\mathbf{u}_{6}=(0,0,-1)$. Note that this arrangement of sites is perfect for a cubic lattice, in general we would expect the angles between the attractions to be not quite perfectly aligned with the angles in the solid phase, These mismatches will tend to destabilise the solid phase so our model is an extreme case; a general arrangement of sites will tend to have a less stable solid phase.

\section{Theory for the fluid phase}

The theory for the fluid phase of particles interacting via a hard-core and directional attractions mediated by sites is well established. Wertheim developed highly accurate perturbation theories for models with one 16 , 17 and two sites.18, 19 By perturbation theory we mean a theory which calculates the difference in free energy between a fluid of particles interacting via the full potential and that of a fluid of particles interacting just via the hard-sphere part of the potential. Chapman, Jackson and Gubbins, by assuming additivity of the effect of the sites on the free energy, generalised the perturbation theory to potentials with more than two sites.20.21 The theory has been extensively tested against the results of computer simulation. 13 . 15,20

Thus, the perturbation theory gives for the Helmholtz free energy per particle of the fluid phase, $a_{f} 20$

$$
\beta a_{f}(\eta, T)=\beta a_{h s}(\eta)+n_{s}\left[\ln X+\frac{1}{2}(1-X)\right],
$$

where $a_{h s}$ is the Helmholtz free energy per particle of a fluid of hard spheres. We use an accurate expression derived from the equation for the pressure of Carnahan and Starling24.25 for $a_{h s}$. The volume fraction $\eta=\rho(\pi / 6) \sigma^{3}$ is a reduced density; $\rho$ is the number density of particles. $\beta=1 / k T$, where $k$ is Boltzmann's constant and $T$ is the temperature. $X$ is the fraction of sites which are not bonded to another site. As all site-site interactions are equivalent the fraction of each type of site which is not bonded is the same. The fraction of sites which are bonded and the fraction which are not bonded must, of course, add up to one. Thus we can simply write down a mass-action equation for $X, 20$

$$
1=X+\rho X^{2} K g_{h s}^{c}(\eta) \exp (\beta \epsilon)
$$

where $g_{h s}^{c}$ is the contact value of pair distribution function of a fluid of hard spheres. It can begbtained from Carnahan and Starling's expression for the pressure of hard spheres using the virial equation.25 The volume of phase space (both translational and orientational coordinates) over which a bond exists is $K, 20$

$$
K=\pi \sigma^{2}\left(r_{c}-\sigma\right)\left(1-\cos \theta_{c}\right)^{2} .
$$

See Refs. 6, 16 21,26 for a detailed discussion and derivation of Eqs. (1) and (5). The rightmost term in Eq. (5) is the fraction of sites which are bonded. This is equal to the fraction of sites which are unbonded and so are available to form a bond, $X$, times the probability of such a site forming a bond. This probability is the density of other sites available for bonding, $\rho X$, times a Boltzmann factor $\exp (\beta \epsilon)$ and integrated over the phase volume for which a bond exists. The factor of $g_{h s}^{c}$ is required at high density to take account of the effect of surrounding particles pushing particles together. As for the free energy, Eq. (4), it is easy to check that, for $n_{s}=2$, it gives the correct low density limit.26 
The mass-action equation, Eq. (5), is a quadratic equation for $X$ and the physical solution is

$$
X=\frac{2}{1+\left[1+4 \rho K g_{h s}^{c} \exp (\beta \epsilon)\right]^{1 / 2}},
$$

Note that Eqs. (5) and (7) are not quite the same as the equivalent equations in Refs. 6, 16, 21, 26. In those references $\exp (\beta \epsilon)$ is replaced by $\exp (\beta \epsilon)-1$. As $\beta \epsilon$ is quite large, five or more, the difference between the two is very small. Also, our $K$ is $4 \pi$ times the $K_{A B}$ of Ref. 20 .

The pressure of the fluid phase, $p_{f}$, can be found by differentiating the free energy, $a_{f}$ :

$$
\begin{aligned}
\beta p_{f} & =\rho^{2}\left(\frac{\partial \beta a_{f}}{\partial \rho}\right) \\
& =\beta p_{h s}+n_{s} \rho^{2}\left(\frac{\partial X}{\partial \rho}\right)\left(\frac{1}{X}-\frac{1}{2}\right)
\end{aligned}
$$

where $p_{h s}$ is the pressure of a fluid of hard spheres, and the derivative of $X$ may be obtained by taking the derivative of Eq. (7). The chemical potential of the fluid phase $\mu_{f}=a_{f}+p_{f} / \rho$. The state of our single component fluid is specified by the ratio of the site energy to the thermal energy, $\beta \epsilon$, and the density, $\rho$, or volume fraction, $\eta$. If there is phase coexistence at any temperature then the two coexisting densities can be obtained from the requirement that the chemical potential and pressure be equal in the two phases. This requirement yields a pair of coupled nonlinear equations which may be solved to give the two coexisting densities.

\subsection{The second virial coefficient}

In experiments on proteins, the strength of the attractions is often assessed by measuring the second virial coefficient, $B_{2}$, via a scattering experiment. The second virial coefficient is equal to the coefficient of the term linear in density in the Helmholtz free energy per particle.25 From Eq. (đ) we see that it will be the sum of the second virial coefficient of hard spheres, $B_{2}^{h s}=(2 \pi / 3) \sigma^{3}$, and a contribution from the sites. This contribution of the sites may be obtained by first setting $g_{h s}^{c}$ to one in Eq. (7); one is its value to lowest order in density. Then we expand out Eq. (7) for $X$ as a density expansion and truncate after the linear term. This truncated density expansion for $X$ is then substituted in Eq. (44), and the result expanded out in density. The linear term is the second virial coefficient. The result is

$$
B_{2}=B_{2}^{h s}-\frac{n_{s}}{2} K \exp (\beta \epsilon)
$$

\section{Theory for the solid phase}

At low temperature, solidification is driven by the attractive interactions, not packing effects as it is with hard spheres27 or with particles with only one or two sites.28 In the solid phase the position of a particle's centre of mass and its orientation are constrained by the need for it to always be in such a position and orientation that none of the bonds it makes with the neighbouring particles are broken. Like hard spheres the particle is constrained by its neighbours but unlike hard spheres the constraint is not only due to the requirement that the particle not overlap with one of its neighbours but also due to the requirement that bonds not be broken,

We will use a cell theory to describe the free energy of the solid phase of our model 29.30 This means we will approximate the energy by the energy when all bonds are formed, the ground-state energy, and the entropy by the logarithm of the phase volume available to a particle when it is constrained to lie in the solid, forming these $n_{s}$ bonds with its neighbours. Vega and Monson 15 have used a cell theory to describe the solid phase of a very similar model, a simple model of water. They avoid a couple of the approximations used here at the cost of not having an analytical free energy. Comparison with the results of computer simulation show 
that their cell theory is quite accurate. Within a cell theory for a solid phase, the Helmholtz free energy per particle, $a_{s}$, is given by

$$
\beta a_{s}=-\ln q_{P}
$$

where $q_{P}$ is the partition function of a single particle trapped in a cage formed by the requirements that all its $n_{s}$ sites bond to neighbouring particles, and that its hard core not overlap with any of these neighbours. In order for these bonds to not be broken the particle must always be within $r_{c}$ of the surrounding particles. This fixes the lattice constant, $a$, at a little less than $r_{c}$. It is a little less as when the particle moves off the lattice site it will be moving towards some of its neighbours and away from others. Thus it can explore regions where it is further than $a$ from some of its neighbours. Of course, a particle cannot move within $\sigma$ of any of its neighbours due to the hard-sphere interaction. Thus the particle can move a distance $a-\sigma$ in the direction of any of its neighbours. The exact value of the maximum lattice constant for which the particle can move about, constrained by the hard-sphere interactions, without breaking any bond, is difficult to estimate; as is the volume available to the centre of mass of the particle.29 Therefore, we approximate the lattice constant $a$ by $r_{c}$ and the volume to which the particle is restricted by $\left(r_{c}-\sigma\right)^{3}$. The requirement that no bonds be broken also severely restricts the orientations of the particle. When a non-axially symmetric particle is free to rotate it explores an angular phase space of $8 \pi^{2}$. However, in the solid its rotations will be restricted to those which are small enough not to violate the requirement that the orientations of its site vectors are within $\theta_{c}$ of the lines joining the centre of the particle with the those of the neighbouring particles. Again the exact value of angular space available to the particle is complex, and it also depends on the position of the particle. We approximate this angular space by assuming that each of the three angular degrees of freedom can vary independently over a range of $2 \theta_{c}$. The normalised angular space available to a particle in the solid phase is then $\left(2 \theta_{c}\right)^{3} / 8 \pi^{2}=\theta_{c}^{3} / \pi^{2}$. The energy per particle is, of course, $-\left(n_{s} / 2\right) \epsilon$, and so the partition function, $q_{P}$, is then just the volume available to the centre of mass of the particle times the angular space available times $\Lambda^{-1} \exp \left[\left(n_{s} / 2\right) \beta \epsilon\right]$, where $\Lambda^{-1}$ is the integral over the momentum degrees of freedom. Thus, we have for $q_{P}$,

$$
q_{P}=\left(r_{c}-\sigma\right)^{3}\left(\frac{\theta_{c}^{3}}{\pi^{2}}\right) \Lambda^{-1} \exp \left(\frac{n_{s}}{2} \beta \epsilon\right)
$$

Inserting this expression for $q_{P}$ into Eq. (11),

$$
\beta a_{s}=-3 \ln \left(\frac{r_{c}}{\sigma}-1\right)-\ln \left(\frac{\theta_{c}^{3}}{\pi^{2}}\right)-\frac{n_{s}}{2} \beta \epsilon=\beta \mu_{s},
$$

where we have neglected a term $\ln \left(\Lambda / \sigma^{3}\right)$. This is the free energy at a lattice constant of $r_{c}$. The maximum possible density of a simple-cubic lattice is when the lattice constant $a=\sigma$, then the density is $\sigma^{-3}$. This density corresponds to a volume fraction $\eta=\pi / 6$. When the lattice constant is $r_{c}$, the density is $r_{c}^{-3}$ and the volume fraction is $(\pi / 6)\left(\sigma / r_{c}\right)^{3}$. On compression, the free energy of solid phase increases very rapidly and diverges when $a=\sigma$.

We are interested in finding coexistence between the solid phase and the fluid phase at low temperature, when our assumption that no bonds are broken in the solid phase will be accurate. Then the pressure at coexistence will be low and the solid will be near the its minimum possible density, $r_{c}^{-3}$. The chemical potential $\mu_{s}=a_{s}+p_{s} / \rho$ where $p_{s}$ is the pressure and $\rho$ is the density. At low pressure $p_{s} / \rho$ contributes a negligible amount to the chemical potential, which enables us to equate $a_{s}$ and $\mu_{s}$ as we have done in Eq. (13). The coexisting fluid density at the fluid-solid transition is then found by equating the chemical potentials in the two phases. The density of the coexisting solid phase, when the temperature is low enough that solidification is driven by the attractive interactions not packing effects, is assumed constant at $r_{c}^{-3}$. Essentially, the requirement that the pressure be equal in the two phases disappears due to the fact that in the solid phase the pressure is very sensitive to density. This is best seen in the schematic diagram of the free energy shown in Fig. 2. The free energy has very narrow minimum at a density of $r_{c}^{-3}$; at higher densities it rapidly increases as the maximum density of the simple-cubic lattice is approached and at lower densities it is much higher as some of the bonds are broken. The pressure $(=$ minus the slope of the free energy with 
respect to the volume) then varies very rapidly. See Fig. 11 of Ref. 15 which shows the results of computer simulations for a similar solid phase; the pressure varies from almost zero to $20 k T / \sigma^{-3}$ over a range in volume fraction of about 0.01 . Now, the more rapid the variation the smaller the displacement in density that is required to vary the pressure of the solid phase to equal that in the fluid phase. By fixing the density of the solid phase to be $r_{c}^{-3}$ and then determining the density of coexisting fluid phase by just equating the chemical potentials in the two phases, we are neglecting this displacement in density.

\section{Phase behaviour}

The only phase transition of hard spheres is a fluid-solid transition 27 Spheres which have only one site and so form only pairs of spheres, also only have a fluid-solid transition.28 When there are two sites, linear chains of particles form, again there is only a fluid-solid transition 31 All these transitions are driven by packing effects, hard spheres pack more efficiently in the solid than in the fluid phase. When there are more than two sites, there is a fluid-fluid transition, according to the free energy of the fluid phase,32 Eq. (四). Also, when there are more than two sites a fluid-solid transition driven by the interactions between sites not the packing of the hard cores, is possible. Indeed at low temperatures the sites will drive the solidification, they will impose the lattice type. It will be the one which maximises the number of sites which can interact. This low temperature solid is well described by the theory of section 4 . We will only plot results at low temperature when this

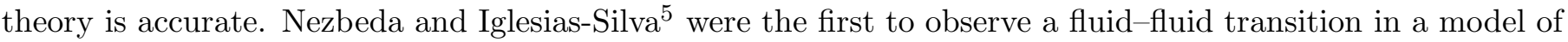
the type we are considering here. Their model has four sites and was developed as a simple model of water.

Figures 3 to 5 are example phase diagrams of four, Figs. 3 and 4, and six site, Fig. 5, model proteins. In each case, as $n_{s}>2$, the fluid phase free energy predicts a transition between a dilute and a dense fluid phase. The values of $\beta \epsilon, \eta$ and $B_{2}$ at the three critical points, $(\beta \epsilon)^{c p}, \eta^{c p}$ and $B_{2}^{c p}$, respectively, are tabulated in Table 1. However, only in Fig. 4 does this transition appear in the equilibrium phase diagram. In Figs. 3 and 5 the fluid-fluid transition is metastable. This means that at equilibrium the transition does not occur, it is preempted by the fluid-solid transition, but if the fluid can be cooled sufficiently far into the two-phase region that it crosses the metastable fluid-fluid coexistence curve then a fluid-fluid transition might be observed. Whether this can be done or not depends on the dynamics of crystallisation.10, 12,33 A metastable fluid-fluid transition was also predicted by Vega and Monson.15 Experiments on globular proteins have found such metastable fluid-fluid transitions 7 . 1 the crystallisation of proteins is often slow, taking several days, which allows the protein solution to be cooled into a region of the phase diagram where the fluid phase separates into two fluid phases of differing densities.

Our toy model of a protein has three parameters: the number of sites, $n_{s}$, the range of the attraction between sites, $r_{c}$, and the angle over which two sites interact, $2 \theta_{c}$. Comparing Figs. 3 and 4 we see that decreasing $\theta_{c}$ destabilises the dense fluid phase (the 'liquid') with respect to the solid phase leaving only a fluid-solid transition in the phase diagram. Comparing Figs. 4 and 5 we see that increasing the number of sites has the same effect. Increasing the range of attraction has a weaker effect, although our theory predicts that increasing the range actually tends to drive a fluid-fluid transition metastable. This is opposite to what is found for isotropic attractions where increasing the range stabilises the dense fluid phase, resulting in the appearance of an equilibrium fluid-fluid transition,

The particles have six degrees of freedom, three translational and three rotational. In order for a particle to form a bond with another particle both its position and orientation must be within small bounds. The distance between the centre of mass of the particle and that of the particle to which it is bonding must lie within the range $\sigma$ to $r_{c}$. The orientation of the site vector $\mathbf{u}_{i}$ must also lie within $\theta_{c}$ of the line joining the two centres of mass. From Eq. (6) we see that in order for a bond to be formed between a pair of particles one of the translational degrees of freedom must be restricted to the small range $r_{c}-\sigma$ (the other two correspond to motion over a sphere of radius $\simeq \sigma$ and so are much less restricted), and two of the orientational degrees of freedom must be restricted (the other one corresponds to rotation about the axis of the bond and is unrestricted). In the solid phase all six degrees of freedom are restricted to small ranges, $\simeq r_{c}-\sigma$ for the 
translational degrees of freedom and $\simeq \theta_{c}$ for the orientational degrees of freedom. However, in the solid phase the particle forms $n_{s}$ bonds. In the fluid phase each bond that forms requires the restriction of one translational and two orientational degrees of freedom. So, in the solid phase when we go from four to six sites then the entropy does not change by much as in both cases all six degrees of freedom are restricted. Our theory, Eq. (13), actually predicts that the entropy is identical for four and for six sites but this is a consequence of the approximations we have made. But of course on adding two extra sites the energy per particle decreases by $\epsilon$. Thus the extra sites reduce the chemical potential of the solid phase, this then coexists with a lower density fluid phase: a fluid phase at a density below the lower of the two fluid-phase densities of the fluid-fluid transition. The fluid-fluid transition is then metastable.

On examining Table 1 we see that neither the critical volume fraction, $\eta^{c p}$, nor the value of the second virial coefficient at the critical temperature, $B_{2}^{c p}$, are sensitive to the value of $\theta_{c}$. However, $\eta^{c p}$ is sensitive and $B_{2}^{c p}$ is very sensitive to the number of sites. Broide et al. estimate that the volume fraction of the globular protein $\gamma$-crystallin in the solution at the critical point is approximately $20 \%$, whereas that of lyzozyme is approximately $15 \%$. To give some idea of what we might expect $\eta^{c p}$ to be, its value in the van-der-Waals fluid is 0.13 . The van-der-Waals fluid is a fluid of hard spheres with a very long-range attraction.25, 28 Both our six-site model and short-range isotropic attractions yield critical volume fractions above 0.13 but our four-site model predicts a lower volume fraction. Thus, the experiments rule out modeling the attractions as just four sites, at least for $\gamma$-crystallin. This is not a surprise, we expect there to be an isotropic attraction present and are neglecting it here purely for simplicity.

As for the second virial coefficient at the critical temperature. It is equal to $-3.5 \sigma^{3}$ for a van-der-Waals fluid. For short-range isotropic attractions it is less negative than this 34 Indeed as the range of an isotropic attraction tends to zero the fluid-fluid transition crosses the fluid-solid transition and becomes a solid-solid transition and the value of the second virial coefficient at the critical point of the solid-solid transition tends to towards its value for hard spheres, $(2 / 3) \pi \sigma^{3}$. Correspondingly, $\eta^{c p}$ tends towards the close-packing volume fraction for hard spheres, $\simeq 0.74$. The second virial coefficient of solutions of globular proteins can be measured via a scattering experiment. Measuring it under conditions near those for which the metastable fluid-fluid transition has its critical point would shed some light on the nature of the interactions. For instance, a large and negative second virial coefficient would rule out isotropic attractions as the dominant cause of the fluid-fluid transition. A small and negative second virial coefficient would however be ambiguous; both short-range isotropic attractions and our six-site model would be consistent with this finding.

Our approximation for the free energy of the solid phase, Eq. (13) is quite crude. We can estimate the accuracy of the phase diagrams we have calculated by replacing our approximation of the lattice constant $a$ by $r_{c}$ which overestimates $a$, by the more conservative estimate $a=\left(r_{c}+\sigma\right) / 2.15$ With this approximation the trends we observe do not change however the range of parameter values over which the fluid-fluid transition is metastable does change. For example, with the parameter values used in Fig. 3 and approximating $a$ by $\left(r_{c}+\sigma\right) / 2$ the fluid-fluid transition is just stable. It is stable for values of $\theta_{c} \geq 0.28$ whereas with the approximation $a=r_{c}$ the fluid-fluid transition is only stable for $\theta_{c} \geq 0.43$. By stable we mean that the critical point of the fluid-fluid transition does not lie within the fluid-solid coexistence region. The approximation $a=\left(r_{c}+\sigma\right) / 2$ also, of course, implies a denser solid phase than $a=r_{c}$.

\section{Conclusion}

A simple model of a globular protein molecule, incorporating highly directional interactions, has been proposed. Example phase diagrams, Figs. 3 to 5, have been calculated. As observed by Vega and Monson 15 highly directional attractions can have phase diagrams which are similar to those of short-range isotropic attractions; there is a fluid-fluid transition but it is metastable. We have also been able to show that if the number of sites is not too large, then directional attractive interactions can induce a stable fluid-fluid transition, see Fig. 4. As far as we are aware this is the first demonstration which shows a fluid-fluid transition driven by directional attractions which is definitely stable with respect to a fluid-solid transition. There have 
been several calculations of fluid-fluid transitions driven entirely by directional attractions with no isotropic attraction present5 13, 14 but only Vega and Monson 15 considered the solid phase.

Clearly, our potential with only directional attractions is as much a caricature of the interaction between globular proteins as is a potential with only a short-range isotropic attraction. It does have one advantage, however, in that, unlike isotropic attractions, the solid phase is not necessarily either face-centred-cubic or hexagonal-close-packed. In our model the lattice is determined by the arrangement of the sites. We chose an arrangement which allowed all four or six sites to form bonds in a simple-cubic lattice but we could equally well choose arrangements of sites which can bond in, say, a body-centred-cubic lattice. Globular proteins form solids with a variety of different solid lattices. If the interaction between globular proteins can be represented without too much error by a pairwise additive potential then the limited amount that we know of interactions between globular proteins suggests that a potential with a hard-core, and both isotropic and directional attractions is the most appropriate one to choose. Such potentials have been used with considerable success to predict the phase behaviour of hydrogen-bonding liquids. 136

Our model does raise one issue: that of differences between the many globular proteins. Previous work 912 on models with isotropic attractions inherently assumes that all globular proteins are alike. This supposition is supported by the presence of metastable fluid-fluid phase transitions for several different globular proteins. However, the ease with which a globular protein crystallises differs enormously from protein to protein. Indeed the fact that some have never been crystallised provides much of the motivation behind the work. If we vary the orientations of the site vectors, $\mathbf{u}_{i}$, then we dramatically change the stability of the solid phase. If the vectors are arranged in way which is not compatible with any solid lattice then at low temperatures there will be no solid phase (except at extremely high osmotic pressures). If in the solid phase not all the bonds can be made but they can in the fluid phase then the fluid will not solidify as this will increase the energy, and at low temperature the energetics dominates. We speculate that the proteins which have resisted efforts to crystallise them may have directional attractions which are not compatible with any solid lattice. If this is so then crystallising them will be all but impossible without minimising the strength of these directional attractions which will act to stabilise the fluid phase.

\section{References}

[1] S. D. Durbin and G. Feher, Ann. Rev. Phys. Chem. 47, 171 (1996).

[2] Protein Interactions, edited by H. Visser (VCH, Weinheim, Germany, 1992)

[3] B. L. Neal, D. Asthagiri, O. D. Velev, A. M. Lenhoff and E. W. Kaler, J. Cryst. Growth. 196, 377 (1999).

[4] See M. N. Garcia-Lisbona, A. Galindo, G. Jackson and A. N. Burgess, Mol. Phys. 93, 57 (1998) and references therein.

[5] I. Nezbeda and G. A. Iglesias-Silva, Mol. Phys. 69, 767 (1990).

[6] R. P. Sear and G. Jackson, Proceedings of the International School of Physics 'Enrico Fermi' Course CXXIX, edited by M. Baus, L. F. Rull and J. P. Ryckaert (Kluwer, Dordrecht, 1995).

[7] M. L. Broide, C. R. Berland, J. Pande, O. O. Ogun and G. B. Benedek, Proc. Nat. Acad. Sci. 88, 5660 (1991).

[8] M. Muschol and F. Rosenberger, J. Chem. Phys. 107, 1953 (1997).

[9] D. Rosenbaum, P. C. Zamora and C. F. Zukoski, Phys. Rev. Lett. 76, 150 (1996).

[10] P. R. ten Wolde and D. Frenkel, Science 277, 1975 (1997).

[11] W. C. K. Poon, Phys. Rev. E 55, 3762 (1997). 
[12] R. P. Sear, J. Chem. Phys, in press; cond-mat/9903301 (http://xxx.lanl.gov/).

[13] D. Ghonasgi and W. G. Chapman, Mol. Phys. 79, 291 (1993).

[14] Y. Duda, C. J. Segura, E. Vakarin, M. F. Holovko and W. G. Chapman, J. Chem. Phys. 108, 9168 (1998).

[15] C. Vega and P. A. Monson, J. Chem. Phys. 109, 9938 (1998).

[16] M. S. Wertheim, J. Stat. Phys. 35, 19 (1984); ibid 35, 34 (1984).

[17] M. S. Wertheim, J. Chem. Phys. 85, 2929 (1986).

[18] M. S. Wertheim, J. Stat. Phys. 42, 459 (1986); ibid 42, 477 (1986).

[19] M. S. Wertheim, J. Chem. Phys. 87, 7323 (1987).

[20] G. Jackson, W. G. Chapman and K. E. Gubbins, Mol. Phys. 65, 1 (1988).

[21] W. G. Chapman, G. Jackson and K. E. Gubbins, Mol. Phys. 65, 1057 (1988).

[22] L. Boltzmann, Lectures on Gas Theory (Dover, New York, 1995).

[23] M. P. Allen and D. J. Tildesley, Computer Simulations of Liquids (Clarendon Press, Oxford, 1987).

[24] N. E. Carnahan and K. E. Starling, J. Chem. Phys. 51, 635 (1969).

[25] J.-P. Hansen, and I. R. McDonald, Theory of Simple Liquids (Academic Press, London, 2nd Edition, 1986).

[26] R. P. Sear and G. Jackson, J. Chem. Phys. 105, 1113 (1996).

[27] W. G. Hoover and F. H. Ree, J. Chem. Phys. 49, 3609 (1968).

[28] R. P. Sear and G. Jackson, J. Chem. Phys. 102, 939 (1995).

[29] R. J. Buehler, R. H. Wentorf, J. O. Hirschfelder and C. Curtiss, J. Chem. Phys. 19, 61 (1951).

[30] R. P. Sear, Mol. Phys. 96, 1013 (1999).

[31] A. Malanoski and P. A. Monson, J. Chem. Phys. 107, 6899 (1997).

[32] Although our model is only defined for $n_{s}$ an even integer, in the free energy of Eq. (承, we can treat $n_{s}$ as a continuous variable. If we do so we find a fluid-fluid transition for $n_{s} \geq 2$. As $n_{s} \rightarrow 2$ the temperature and density at the critical point of the fluid-fluid transition both tend towards zero. 14

[33] P. G. Debenedetti, Metastable Liquids (Princeton University Press, Princeton, 1996).

[34] M. H. J. Hagen and D. Frenkel, J. Chem. Phys. 101, 4093 (1994).

[35] P. Bolhuis, M. Hagen and D. Frenkel, Phys. Rev. E 504880 (1994).

[36] A. Gil-Villegas, A. Galindo, P. J. Whitehead, S. J. Mills, G. Jackson and A. N. Burgess, J. Chem. Phys. 106, 4168 (1997). 


\begin{tabular}{|c|c|c|c|}
\hline Figure & $(\beta \epsilon)^{c p}$ & $\eta^{c p}$ & $B_{2}^{c p}$ \\
\hline 3 & 10.24 & 0.090 & $-15.5 \sigma^{3}$ \\
4 & 8.64 & 0.090 & $-15.5 \sigma^{3}$ \\
5 & 7.18 & 0.154 & $-4.0 \sigma^{3}$ \\
\hline
\end{tabular}

Table 1. The ratio of the interaction site energy to the thermal energy at the critical temperature, $(\beta \epsilon)^{c p}$, the second virial coefficient at the critical temperature, $B_{2}^{c p}$, and the volume fraction at the critical point, $\eta^{c p}$, are tabulated for the parameters used in Figs. 3 to 5 . Both $\eta^{c p}$ and $B_{2}^{c p}$ are identical for the parameters of Figs. 3 and 4 as within our theory for the fluid phase changing either $\theta_{c}$ or $r_{c}$ simply changes the temperature scale of the phase behaviour.

Fig. 1. A schematic diagram of our model of a globular protein. The circle represents the hard core of the particle and the shaded circles represent three of the four conical attraction sites (the fourth is on the other side of the sphere).

Fig. 2 A schematic diagram of the free energy of coexisting fluid and solid phases. The solid curves are the free energy per particle (over $k T$ ). The dashed line joins the coexisting phases.

Fig. 3. The phase diagram of our model of a globular protein. The number of sites $n_{s}=4, r_{c}=1.05 \sigma$ and $\theta_{c}=0.3$ radians or about $17^{\circ}$. The solid curves separate the one and two-phase regions. The letters $\mathrm{F}, \mathrm{S}$ and 2 denote the regions of the phase space occupied by the fluid phase, the solid phase and coexistence between the fluid and solid phases. The dashed curve is the coexistence curve for a metastable fluid-fluid transition.

Fig. 4. The phase diagram of our model of a globular protein. The number of sites $n_{s}=4, r_{c}=1.05 \sigma$ and $\theta_{c}=0.45$ radians or about $26^{\circ}$. The solid curves separate the one and two-phase regions. The letters $\mathrm{F}, \mathrm{S}$ and 2 denote the regions of the phase space occupied by the fluid phase, the solid phase and coexistence between two phases, either two fluid phases or the fluid and solid phases. The dashed curves are the metastable continuation of the fluid-fluid coexistence curve, and the dotted curve is the metastable continuation of the high-density-fluid-solid coexistence curve. The latter terminates when it touches the spinodal of the fluid-fluid transition.

Fig. 5. The phase diagram of our model of a globular protein. The number of sites $n_{s}=6, r_{c}=1.05 \sigma$ and $\theta_{c}=0.45$ radians or about $26^{\circ}$. The solid curves separate the one and two-phase regions. The letters $\mathrm{F}, \mathrm{S}$ and 2 denote the regions of the phase space occupied by the fluid phase, the solid phase and coexistence between the fluid and solid phases. The dashed curve is the coexistence curve for a metastable fluid-fluid transition. 


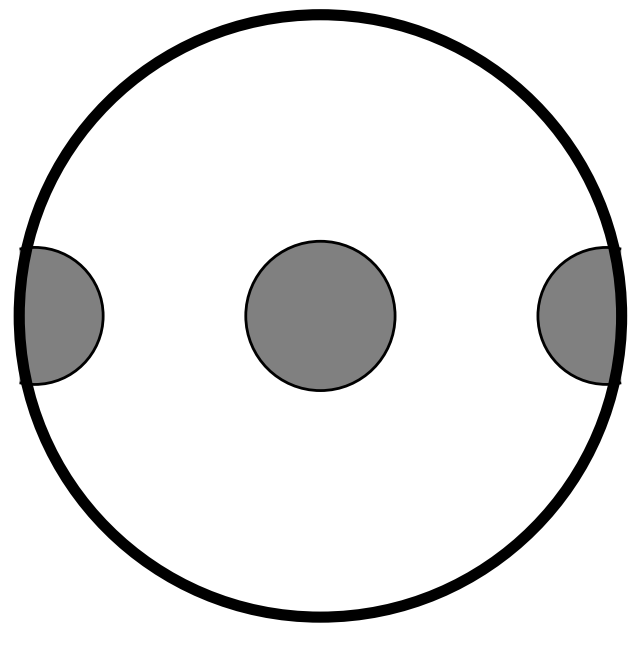




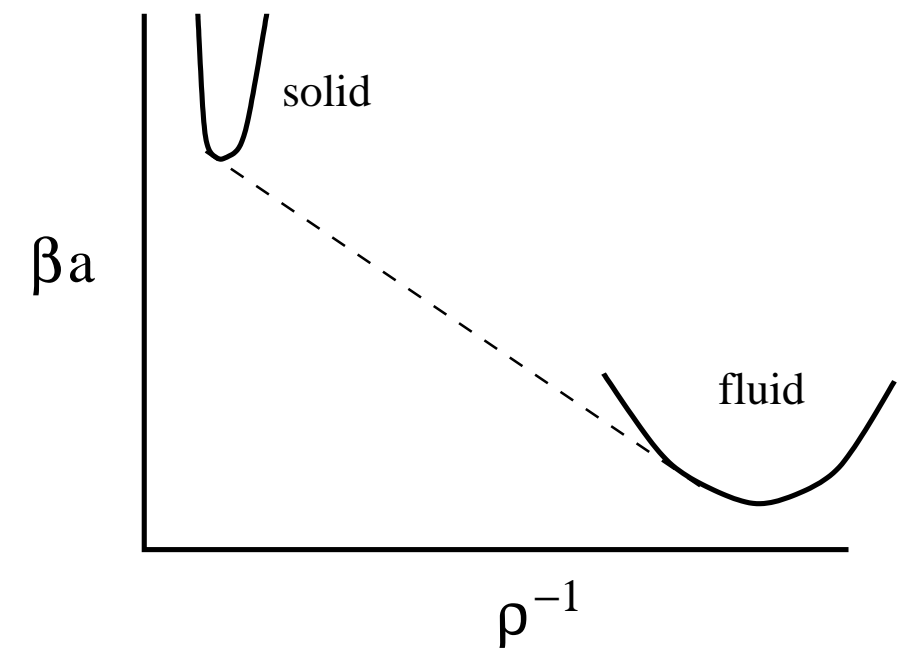




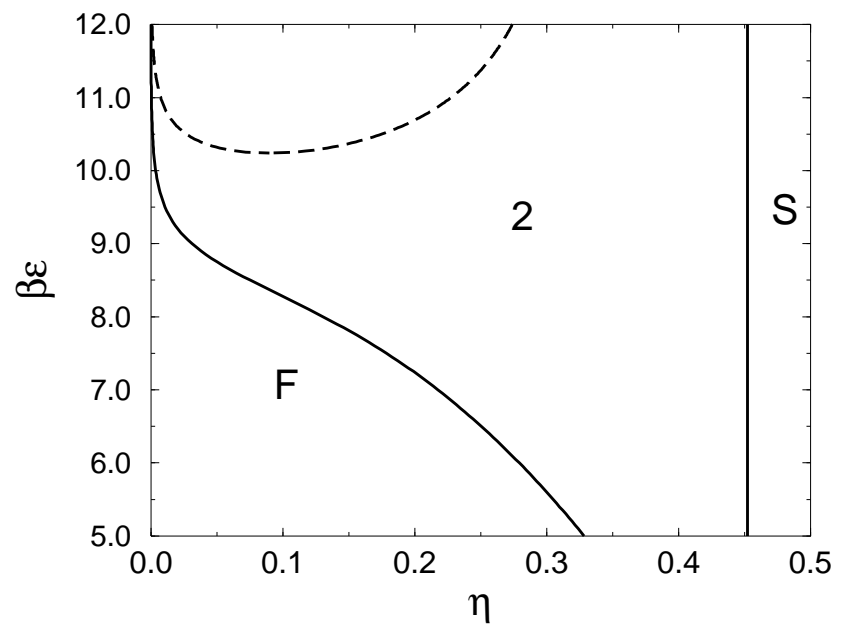




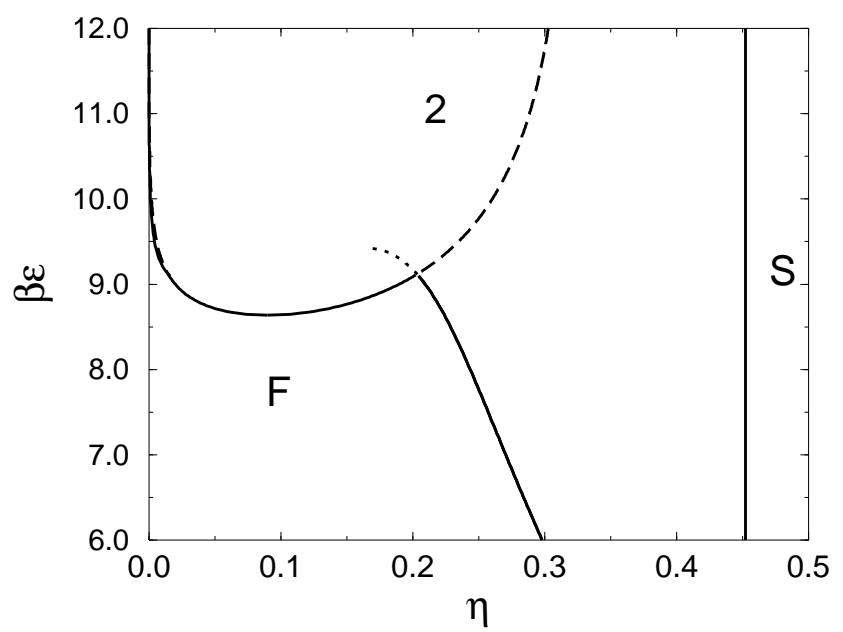




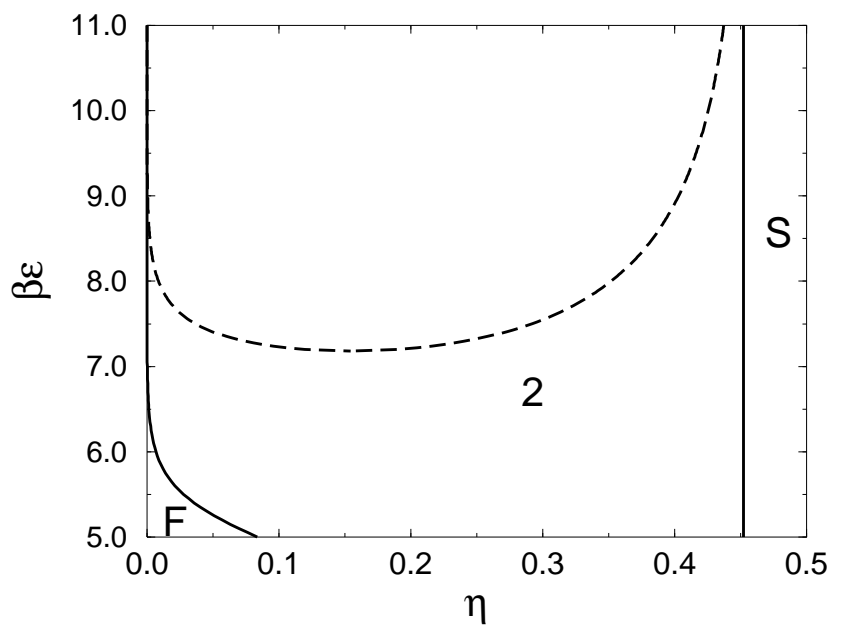

